\title{
Hacia una nueva Concepción de la Seguridad Europea
}

\begin{abstract}
$\therefore \mathrm{L} A$ I $\mathrm{N}$ J $\circ \mathrm{x}$ E es investigador de l'Ecole Pralique des Hantes Eludes (Sorbonne), del Centre d'Eludes ie Politique Elrangire, de Paris, y profesor del Instituto de Estudios Intornacionales de la Ĺniversiciad de Chile. Este artículo está basado en una conferencia dictada en cl Salón de Fionor de la Lniversidad de Chile, el 12 de diciembre de 1966.
\end{abstract}

Dentro de los problemas de estrategia militar, ya sea nuclear a clásica, que se discuten en Europa, está implicada toda una política de orden mundial. A mi parecer, el primer objetivo político de una nación o de un grupo de naciones es su propia conservación y el mejoramiento de las condiciones de vida de su población. Ahora bien, el cuadro geográfico y político dentro del cual un poder se esfuerza por conservarse y progresar, delimita también el territorio que se propone defender contra el mundo exterior. Esta es la razón por la cual la manera como Europa definirá las condiciones de su sezuridad en el momento de la desaparición de la cortina de hierro, reflejará su voluntad de coordinación política y económica; más bien dicho, su interés por actuar en el munclo como una entidad política y económica particular; lo que, evidentemente, concierne, y muy de cerca, al porvenir ry desarrollo de los países del tercer mundo. Todos sabemos, en efecto, que no cs el aumento de la ayuda financiera lo que podrá resolver el problema global del subdesarrollo, sino una voluntad política que llegue a modificar las condiciones de la acumulación de capital en los paises subdesarrollados, mediante la estabilización de los términos del intercambio.

$\mathrm{Se}$ admite, generalmente, que estamos viviendo el termino de la guerra fría, pero hay que precisar que se habla del fin de la guerra fria entre Estados Unidos y la Unión Soviética, lo que trae consigo una modificación considerable de la situación europea, cuyas consecuencias todaría no todos los europeos están dispuestos a aceptar.

In Asia, por el contrario, si se hubiera mantenido la unión entre China y la Unión Sovićtica, estaríamos en plena guerra fría, tal como en los momentos más clramáticos de la guerra de Corea: Como -1l lit attualidad I sistema mundial se divide en, por lo menos, tres 
centros de primera importancia, creo que estamos viviendo, a la vez, el fin de la guerra fría bipolarizada y el fin de la coexistencia pacífica bipolarizada. Esto complica las perspectivas de las relaciones internacionales, y esta complicación permite a las pequeñas y me. dianas potencias, en especial las europeas, hacer oir su voz sobre sus problemas particulares.

De todas maneras, Europa, en el momento actual, se presenta como un lugar del mundo donde impera la coexistencia pacífica y donde, por lo tanto, el problema de la seguridad aparece con caracteres completamente nuevos.

Durante todo el periodo anterior, la seguridad de Europa Occidental no pudo ser afianzada sino mediante el empleo de recursos militares que, por definición, ponían en peligro la seguridad de la Europa Oriental. Hoy clía, por el contrario, vivimos el comienzo de una época en la que la seguridad de Europa Occidental y la seguridad de Europa Oriental ya no aparecen como términos antagónicos, sino complementarios. Esto se debe principalmente a las transformaciones del sistema mundial.

Para encarar esta cuestión de manera concreta no podemos limitarnos a hacer un resumen de la lógica de un sistema de clos o tres potencias: La historia del paso de dos a tres es lo único que puede aclararnos la situación actual. Esta historia está intimamente ligada a las decisiones políticas de los líderes en dos campos fundamentales: 1) La posesión de armas nucleares, y 2) La definición del espacio económico óptimo (Bloque económico socialista, con o sin China; "Transatlantismo" económico o Europa Occidental autónoma - Europa hasta los Urales).

Hay intereses materiales concreto: en juego, ya se trate de capacidad de producción o de capacidad de destrucción (lo que implica la producción de armamentos).

En todos estos aspectos materiales, Europa, que es la parte desarrollada del mundo, tanto por su potencial inclustrial como por la ex:ensión de su mercado, ha desempeñado un papel mitad pasivo, mitad activo, muy importante.

Me limitaré aquí a enfocar, primerạmente, a través de la historia de los sistemas estratégicos y de los sistemas de armamentos, una parte de la historia de la despolarización en Europa.

Esta crónica nos llevará en seguida' a hacer un análisis del momento actual y de las posibilidades de exito de un nuevo sistema de seguridad, no ya para una Europa diviclida en bloques sino para una Europa cuyas dos partes se entienden. 
DepolarizactóN

Sc acostumbra a distinguir cuatro períolos en la listoria de la confrontación estratégica cntre el Este y el Oeste clespués de la última gran guerra. Consideramos los años que van desde 1945 a 1965.

\section{Priner Período}

Va descle 1945 hasta alrededor de 1950. Se le puede definir asi: Los Estados Unidos tienen el monopolio de la bomba atómica, aunque sin abundancia, porque en esta época hay escasez de materiales fisibles. Hay superioridad americana en bombarderos estratégicos y una aplastante superioridad soriética en armas convencionales. La Unión Soviética cuenta también con un importante caza.

Hay, por lo tanto, superioridad estratégica americana debido al arma atómica, pero esta superioridad no es adecuada para luchar contra ciertas tentativas soviéticas o inspiradas por los soviéticos. Las acciones favorables a los soviéticos están constituidas principalmente por ofensivas políticas destinadas a contrapesar su inferioridad nuclear con la lucha, por así decir, cuerpo a cuerpo.

Stalin actuó siempre con extremada prudencia y no pensó seguramente nunca en una gran invasión, ya que ésta habría sido muy vulnerable a una respuesta nuclcar americana. Sin embargo, como en esta época Europa es una especie de rehén del ejército ruso, la superioridacl nuclear no puede ser utilizada de manera ofensiva $y$ hasta las contraofensivas occidentales son prudentes. Recordemos que los intercambios de golpes que caracterizan este período en Europa son, mirados desde cierta distancia, relativamente moderados y equilibrados.

Entonces, lo mismo que hoy, el confrontamiento guerrero se produce en el Asia.

En 1947 la guerra civil griega dio a Truman la ocasión de lanzar una doctrina americana intervencionista, pero, precisamente, su única aplicación en Europa fue el conflicto griego.

En 1948 la Unión Soriética responde a la división económica de Alemania con el bloqueo no militar.

Al bloquen de Berlín los americanos responden con otra medida no militar: el puente aéreo. El año 1948 marca también la fecha de la instalación de un régimen comunista en Praga. Pero la Unión Soviética pierde ese mismo añ:s el control de Y'ugoslasia, hecho que pa- 
rece intencionado y que tendrá como consecuencia la derrota de la izquierda en Grecia. En 1949 nace la Alianza del Atlántico y en 1950, después del comienzo de la guerra de Corea, la otAN (Organización del Tratado del Atlántico Norte).

Al final de este período, se puede hablar de una consolidación pacifica en Europa y de la fijación de Asia como teatro de operaciones militares.

\section{SECUNDO PERTíno}

Puede ser llamado el período del 50, y se extiende desde 1950 hasta 1957. Se inicia con el fin del monopolio nuclear norteamericano y está caracterizado por la aparición en 1953 de las armas termonucleares, casi al mismo tiempo, en Estados Unidos y en la Unión Soviética. El primer Sputnik es lanzado en 1957. El equilibrio estratégico de este período permanece esencialmente dominado por la existencia del SAC norteamericano (Strategic Air Command -Comando Aéreo Estratégico). Los Estados Unidos, que han rodeado a la Unión Soviética de una serie de bases aéreas estratégicas, no son aún realmente vulnerables, ya que la capacidad atómica soviética y sus medios de transporte son reducidos; entretanto, los Estados Unidos comienzan a conocer la abundancia en materiales nucleares. El espacio europeo es apreciado en Occidente por su capacidad de ofrecer bases para el sAC, próximas al territorio soviético; y en la Unión Soviética, por el papel de rehén que Europa continúa desempeñando, bajo una virtual amenaza de invasión.

Recordemos los acontecimientos que jalonan este período: En 1951, termina la guerra de Corea. En 1953, muere Stalin. En 1954, termina la guerra de Indochina. En 1955, se rearma Alemania y se crea el Pacto de Varsovia. En I956, se producen el octubre polaco, la rebelión húngara y la expedición a Suez: éstas son las primeras manifestaciones del debilitamiento de las alianzas. En 1957, el lanzamiento del primer Sputnik.

La prudencia que observan los dos Grandes es aún más marcada que en el período anterior, actitud que hay que relacionar con el comienzo de una política de disuasión nuclear recíproca. Es la détente anunciada ya por Stalin en la apertura de las negociaciones sobre el tratado del estado austriaco. Sin embargo, el acontecimiento político más importante es el rearme.de la República Federal, obte. nido a pesar de que el estatuto de Alemania aún no ha sido resuelto, y en un momento en que las más grandes tensiones se han aflojado.

Esto nos obliga a meditar sobre la relativa importancia que tienen para Occidente los argumentos militares y los argumentos políticos: 
Durante este segundo período (de 1950 a 1957) la teoría del rollback y, en Alemania, la idea de que la reunificación alemana a favor de Occidente podía efectuarse bajo la presión de la superioridad militar, han sido ideas dominantes.

Ya se haya tratado de un mito, ya de un complemento necesario a la integración de Alemania Occidental a la vida política democrática liberal y a la economía occidental, el ejército alemán, hoy día el más poderoso de Europa después del soviético, es la consecuencia de un dispositivo militar que debía servir a una ofensiva política que la historia ha desplazado. El rearme alemán, instrumento tardio de la seguridad europea en los años 50 , se ha convertido hoy en el problema principal de la reunificación de Europa y la segưridad europea. Esta no es sino una más de las numerosas paradojas de la historia.

\section{Tercer Período}

Llegamos, a continuación, a un tercer periodo: el que inicia la era de la estrategia de los cohetes. Este período se sitúa entre 1957 y 1962. Como fecha límite podemos elegir la declaración de Kruschev, en agosto de 1957, anuncianclo la preparación de los cohékes intercontinentales por la Unión Soviética, o el lanzamiento del Sputnik, en septiembre de 1957.

Sin embargo, podemos alejar esta fecha hasta febrero de 1959, momento en que los técnicos soviéticos realizan el priner ensayo de lanzamiento de un cohete de largo alcance. Fue lanzado desde orillas del mar Caspio hasta el mar de China, con una precisión de unos pocos kilómetros alrededor del objetivo. Este es un acierto de gran importancia, considerando que la distancia es de cerca de 10.000 kílómetros y si se supone que estos cohetes están equipados con bombas temonuclearcs de un poderio de decenas de megatones de INT. En realidad, lo que modificó las mentalidades de los responsables y las opiniones acerca de la naturaleza de la cliscusión sobre programas de armamento, fue el lanzamiento del Sputnik. Esto no cambió la relación de fuerzas, a menos que se entiencla por ella la idea que se formaron ciertos proiagonistas de está relación, lo que, por lo demás, es perfectamente lícito.

Sen como fuere, a partir clel ensayo soviético de 1959, los europeos se encuentran a seis o siete minutos de vuelo de un cohete soviético y los Estados Unidos a treinta o cuarenta minutos. La diferencia no es tan importante como el hecho de que, por primera vez, el espacio $\therefore$ igráfico norteamericano aparece realmente vilnerable. 
El 29 de abril de 1959 el señor Christian Herter responde a una pregunta del senador Wayne Morse acerca de las concliciones en que podrían ser utilizaclas las armas nucleares, de la siguiente manera: "no antes de estar nosotros en peligro".

El comienzo de este nuevo período se caracteriza por un segundo orden de hechos que concierne directamente a Europa; ante todo, hay que decir que, entre 1957 y 1967, Europa comienza a disponer de los aparatos llamados "tácticos". Estos llevan una cabeza nuclear de un potencial parecido al de la bomba de Hiroshima, y' están destinados a ser utilizados en un eventual campo de batalla europeo, para contrapesar la superioridad soviética clásica.

Por otra parte, en septiembre de 1958, se hacen explotar, en el desierto de Nevada, unos obuses atómicos de muy poco poderío - se les llamó "granadas atómicas"- y de pequeño tamaño, lo que hace posible la nuclearización de la artillería.

Estas armas colman el intervalo que existía entre las armas clásicas de explosivo quimico y las armas nucleares de poder megatónico. Esta es la base tecnológica de la estrategia de la escalada, sobre la cual hablaremos más adelante.

Un tercer acontecimiento de este período es la adopción del sistema de los coletes Polaris en submarinos nucleares, propuesta por el Almirante Rickover. Esta decisión no hizo sentir sus efectos sino mís tarde, pero las implicaciones políticas, que ya podian preverse, modifícaron la reflexión política-estratégica de la Alianza Atlántica desde ese mismo momento.

Tres hechos revolucionatios en el progreso de la lecrología de los armamentos fueron entonces de gran importancia para Europa:

1) Cohetes intercontinentales y fin de la invulnerabilidad del territorio de los Estados Uniclos.

2) Miniaturización y posibilidacl de escalada insensible; partiendo del más pequeño inciclente hasta llegax al "espasmo" nuclear.

3) Submarinos nucleares provistos de* cohetes nuclearcs, y' ubicuidad de la amenaza de represalias.

A comienzos del periodo de 1958-62, Ta superioridad estratégica norteamericana se mantiene por la existencia del sac, que representa, por su tamaño, un poder de destrucción considerable, compiementado por algunos cohetes de la primera generación. Al final de este periodo, nos encontramos en plena estrategia de cohetes, pero la reflexión sobre la estrategia de los cohetes de la segunda gencración, de antemam previsible, modifica la atmósfera política durante todo el período.

Lo esencial de la nueva estrategia norteamericana fue anunciado, en 1959, en dos publicaciones, ambas de gran importuncia. 


\section{ESTUDIOS INTER NACIONALES}

En un artículo titulado, "The delicate balance of terror", publicado en Foreign Affairs, en enero de 1959, Albert Wohlstetter, de Ia Rand Corporation, establece:

1) La idea bastante simple de que no puede haber disuasión si no se cuenta con una fuerza capaz de infligir represalias inmediatamente después del ataque del enemigo a quien queremos disuadir.

2) La otra base lógica de la futura estrategia norteamericana consiste en otra idea bastante simple: si anteriormente los Estados Unidos podian disuadir mediante la amenaza brutal de represalias masivas, ahora, con el término clel monopolio norteamericano y la vulnerabilidad del territorio de los Estados Unidos, la amenaza deja de parecer verosímil. Era necesario que la opción si o No fuera reemplazada por cierta flexibilidad, pero contando con la abundancia de medios. Los Estados Unidos podrían pretender, mediante la racionalización de sus inversiones en el capítulo de los armamentos, llegar al dominio de todos los grados cle violencia por la superioridad material en cada tramo de una gradación de escalada, y también la superioridad cuantitativa en el nivel final del "espasmo".

En una obra aparecida en 1959, The Uncertain Trumpet, el general Maxwell Taylor, al admitir que las fuerzas de disuasión norteamericanas, en razón de la existencia de fuerzas soviéticas de represalia, debían arriesgarse solamente en la defensa de posiciones absolutamente esenciales, proponía definir la guerra general como un intercambio de golpes nucleares entre los Estados Unidos y la Unión Soviética, reservando el término "guerra limitada", por lo tanto, para designar todas las otras operaciones militares "en las que se utilizara sobre todo el armamento ciásico, aunque sea necesario conservar la posibilidad de usar las armas atómicas en los raros casos en que su empleo corresponda al interés nacional".

El punto de vista del general Taylor puede justificarse gracias a la asimetría de las situaciones en el Este y en el Oeste, ya que la Unión Soviética podría atacar a Europa sin atacar a los Estados Unidos; en cambio América no podría defender a Europa sin atacar a la Unión Soviética.

Si el medio de equilibrio continúa siendo la aviación portadora de la bomba, a la feliz seguridad de la época anterior sucede el temor justificado de ver a los soviéticos modificar el equilibrio a su favor gracias a la tecnología de los cohetes, y adquirir con ello una superioridad tanto cualitativa como cuantitativa sobre los Estados Unidos (missile gap).

Los augurios pesimistas en los Estados Unidos vienen del thecho de que se pensó que los soviéticos, después de sus ensayos, eran capaces 
de construir cohetes intercontinentales en seric, siendo que en realidad no construyen sino un pequeño número, esforzándose más bien en aumentar el poder de las cargas termonucleares liasta llegar a las decenas de megatones; por otro lado, en Europa despliegan cierto número de inbar (Intermediate Range Ballistic Missiles: Proyectiles balísticos de Alcance Intermedio), destinados a matenerla en el papel de rehén estratégico. Es la época en que se ha dispuesto en Europa de los IRBM (15 Jupilcrs en Turquia, 30 Thors en Italia, 60 Thors en Gran Bretaña. Son coletes "de la primera generación", con tiempo de alerta largo y colocados en sitios no protegidos). Se hace presión también sobre Trancia para que los acepte en su territorio. El gobierno de la Iv República alarga su respuesta. La v dará una respuesta negativa, después que los norteamericanos se niegan a aceptar que cl país de acogida comparta la decisión de emplearlos.

El missile gap que, en 1958, se temía para 1960 ó 1961 y, luego, en 1959, para los años 1962-1963, no se ha producido nunca en realidad. Pero los temores de este clesequilibrio han servido de argumento, por un lado, para la aceleración de los programas de investigación y desarrollo en el clominio de los cohetes, y' por otro, para la elaboración de todo un cuerpo de doctrinas estratégicas nuevas basado en la su. perioridad numérica. Esto fue hecho principalmente en la Rand Corporation $y$ en Harvard por científicos y estrategas civiles que se enconron con poder gracias a la elección del Presidente Kennedy.

El límite final cle este período de urastornos es el año 1962. Este es el año en que el Grand Design cle Kennedy es expuesto en forma clara y simple: es necesario que el Presidente de los Estados Unidos pueda escoger entre varias alternativas. La prueba del sistema o, más bien, de los hombres del equipo que se habían dado la tarea de reformar la administración en este sentido, es la crisis cubana de octubre. Esta crisis también tiene cierta importancia cuando se reflexiona sobre la seguridad europea.

La "crisis de los misiles" es, por cierto, un hecho significativo, aunque ciertamente no a causa del peligro de guerra nuclear inminente que se quería ver en Estados Unidos y, probablemente, también en la Unión Soviética. Desde este punto de vista, creo que podemos decir, parafraseando al Presiclente De Gaulle, en la época del golpe de estado de los generales de Argelia, que la situación era muy grave, pero no seria, ya que no habia nada en el sistema mundial de la época que pudiera llevar a un conflicto entre las dos superpotencias.

Hoy día, menos que en ningún otro momento de la historia de la humanidad, la decisión de comenzar las hostilidades podría nacer de un nuevo despliegue de este tipo, puesto que los mievos despliegues 
de cohetes no tiener nada de una amenaza de invasión y, por lo demás, son moneda corriente en la competencia de prototipos, casi estructural, que se ha iniciado en las economías industriales desarrolladas. La importancia de la crisis radica, pues, en sus implicaciones politicas.

Es evidente que el desarrollo de la crisis, el papel que asumió la Unión Soviética en relación a Castro, la estrategia política de defensa de las conquistas del campo socialista, implicada en el acto de retirar los cohetes soviéticos sin recibir ninguna garantía de parte de los Estados Unidos; todo esto ha tenido una importancia determinante en la evolución acelerada del conflicto chino-soviético.

Por otra parte, si Francia acordó su pleno apoyo a la causa de Estados Unidos, ya que Paris consideraba aceptable que el pueblo norteamericano se sintiera afectado en su sensibilidad política y en lo esencial de sus intereses nacionales, esto no impidió que surgieran conclusiones muy particulares sobre los peligros teóricos que la integración de la OTAN hacía correr a los aliados europeos en caso de crisis aguda fuera de la zona comprendida en el Tratado del Atlántico Norte. Se advirtió que el dispositivo norteamericano habia sido puesto en estado de alerta en Europa, sin que estas medidas hubieran sido objeto de discusión, ni menos de acuerdo previo. El Presidente de la República Francesa ha debido, sin duda, referirse a esto en la alusión a la crisis cubana que hizo en una de sus últimas conferencias de prensa.

Después de 1962, según la expresión utilizada por el general Gallois en un reciente articulo, se entra verdaderamente en la segunda otan, esperada desde 1959.

\section{Guarto Periodo}

El cuarto período es aún el período actual. Va desde 1962 a 1965 , en todo caso, pero el año 19605 constituye tal vez un nuevo momento, sobre todo a causa de la intervención masiva de los Estados Unidos en el Vietnam y del significado de la segunda explosión atómica china.

1. El despliegue de submarinos portadores de armas nucleares es la realización de una nueva forma de encierro reciproco en los mares, por intermedio de armas casi imposibles de detectar y, por lo tanto, de destruir simultáneamente.

2. El desarrollo de los ICBM protegidos (Intercontinental Ballistic Missiles - Proyectiles Intercontinentales Teledirigidos) hace general la relativa invulnerabilidad de las fuerzas nucleares y conduce a una posición de disuasión reciproca casi absoluta. El interés geográfico 
se concentra entonces en las bases para submarinos (Cran Bretaña,

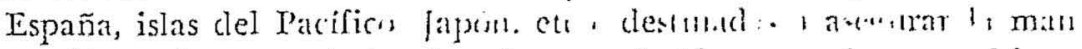
tención y el reposo de las dotaciones, más bien que el reaprovisiona. miento, ya que los submarinos nucleares pueden navegar durante períodos muy largos sin necesidad di realu-tecesc de combustible. En otras palabras, la importancia del espacio europeo la disminuido notablemente en la estrategia general de los dos gigantes nucleares, $y$, por otra parte, las probabilidades de que uno de cllos intervenga en favor de algún país aliado, en caso de presión o ataque, han disminuido igualmente a causa de los riesgos de represalias.

Esto ha sido perfectamente bien comprendido por los europeos de ambos bloques, pero no todos han sacado las mismas conclusiones sobre lo que debe hacerse para afianzar su seguriclad. :Deberán reafirmar formalmente la intima unión de los dispositivos de los países aliados con el lider? $O$, al contrario, tomando conciencia de que la intervención del líder a favor cle un aliado más pequeño puede conducir más bien a la guerra que a la disuasión, en la configuración actual de los sistemas armados, preferir un acercamiento puramente político? Comprendemos que este asunto trae consigo una serie de problemas políticos muy importantes, los que no pueden ser abordados sin entrar en el corazón del problema estratégico y de los nuevos conceptos norteamericanos.

\section{Costo - EFICACIA}

Con el equipo del Presidente Kennedy, tras el secretario de Defensa, el señor Robert McNamara, todo un grupo de lógicos-estrategos se apoderó del Pentigono, tomanclo a su cargo la racionalización de los programas de inversión y la elaboración de una estrategia adecuada a una utilización real, beneficiosa, de los sistemas de armas. Un procedimiento de evaluación de costos y de cficacia, y de costo-eficacia de cada posible sistema de armas permite comparaciones y prepara las decisiones del ejecutivo. Estas decisiones se toman dentro del cuadro de un plan quinquenal reconstituido cada año, lo que da al Pentágono una libertad para negociar que sobrepasa la duración del presupuesto y, aún más, la duración de un Congreso. Este es el único organismo de planificación pública de los Estados Unidos.

\section{ArMs - CoNtrol.}

En el periodo kennediano, la expresión más escuchada y la más rica en significado es arms control, que se puedr tr iscir ' c.' "llir : 
por: "dominio o control de los armamentos". Una cantidad de conceptos diferentes se esconden bajo este slogan:

El arms control, en el sentido más usual, es el "dominio bilateral de los armamentos". Con este sentido aparece en el título de la Arms Control and Disarmament Agency, agencia especialmente creada en el Departamento de Estado para negociar sobre asuntos de reducción o de estabilización de armamentos, o sobre el clesarme. Pero a la sazón, bajo una atmósfera enteramente enderezada hacia la preparación de nuevos sistemas de armas, la Agencia, por sobre los acuerdos puramente formales relacionados con la idea de un desarme general y completo, se orientó más bien hacia el estudio de posibilidades de acuerdos parciales con los rusos -acuerdos limitados desde el punto de vista de los armamentos y acuerdos limitados geozráficamente. Por ejemplo, desde 1962 ó 1963 existe por lo menos una negociación ruso-norteamericana a propósito de un acuerdo regional en Europa (reducción a paridad de las armas clásicas estacionadas en Europa). Este tipo de proyecto, que continúa en el tapete, podria haber conducido a un acuerdo si no se hubiera producido la guerra de Vietnam.

Pero la expresión arms control se aplica, en realidad, sobre todo a los diferentes aspectos de un dominio unilateral de los armamentos, es decir, al dominio de los mismos norteamericanos sobre sus propios programas de armamentos. Este control se había vueito incierto bajo el gobierno de Eisenhower.

En este orden de significados, el control de los armamentos es, ante todo, el dominio cle las inversiones en armamentos, factor bísico para la preparación de una estrategia. Esto significa, por una parte, que las decisiones tomadas sobre la base cle los estudios de costo-eficacia pueclen tener en cuenta una evaluación centralizada de las necesidades, más que las necesidades de cada una de las tres armas. De esta manera, el equipo de McNamara esturo en 1963 en disposición de establecer que las estimaciones de niveles de efectivos clásicos soviéticos habían sido exageradas artificialmente por los responsables del Antiguo Régimen, guiados por razones de negociación presupuestaria. Se calcula desde entonces en 80 y no en 175 el número de divisiones soviéticas. Este "descubrimiento" permite considerar. el siguiente objetivo estratégico: llegar a equilibrar las fuerzas clásicas soviéticas con fuerzas clásicas occiclentales en Europa; de ahí nació el slogan clel aumento del armamento convencional de los europeos (more conventional build-up), el que conoció muy poco éxito.

El arms control es, además, el dominio del sistema operacional de la defensa; debe eliminarse cualquier obstáculo que puecla interpo- 
nerse a una decisión estratégica centralizada, única apta para administrar el arma nuclear, ya se trate de la política o la diplomacia de un estado, ya de la presión ejercida sobre el Departamento de Defensa por alguna de las tres armas. El Pentágono se propone guardar bajo llave las armas nucleares y entregar la llave al Presidente de los EE. UU. Con esto termina la asignación de las armas nucleares a escala divisionaria sobre el teatro europeo.

El arms control es, por último, el dominio de las condiciones de empleo del arma nuclear, es clecir, la flexibilidad, la substitución cle la opción brutal de la época anterior (gonno go) por una serie de opciones posibles en la gradación de la escalada de armas, de las cuales los Estados Unidos poseen, en realidad, el abanico completo. Pero esta doctrina de flexibilidad es extremadamente ambigua para los aliados, no solamente porque puede implicar irresolución o aventurerismo sino, sobre todo, a mi parecer, porque se ha desarrollado en un cuaclro conceptual en el que se evapora la noción de decisión frente a la relación estadística de las fuerzas en juego. Trataré de desarrollar aquí esta idea, haciendo algunas consideraciones sobre la introducción a la escalada, de Herman Kahn, en su libro On Escalation.

\section{ESCALADA}

En esta obra Herman Kahn describe una gradación de escalada que, sezún él, no está destinada a crear categorias sino constituye más bien un "instrumento metafórico". Es considerada como la ordenación lincal del crecimiento de los niveles de intensidad de las crisis.

En su sistema H. Kahn emplea muchas palabras, pero se niega a elaborar conceptos. Sin embargo, toda la formulación de la gradación de escalada se basa en un concepto estaclístico bien disfrazildo, el aumento del uso de medios violentos, es decir, del consumo de vidas humanas $y^{\prime}$ armamentos. Los escalones no son mostrados en esta perspectiva sino para inclicar que hay posibles descansos, en los momentos escogidos por el más fuerte para eventuales negociaciones.

Por último, la mayor ambigüedad, desde el punto de vista de una teoría de la escalada, si hubiera tal, es la siguiente:

Al definir un escalón, el autor se coloca, según el caso, desde el punto de vista de la clecisión de $A$, o desde el punto de vista de la dialéctica de las decisiones $A / B$. Hay, por lo tanto, dos teorías virtualmente presentes. La primcra consiclera cacla escalón como una decisión; la escala o gradación correspondería, en este caso, a un esquema para la claboración de una sccuencia de decisiones. La segunda 
consideraría cada esćalón como una pareja de clecisiones, un conjun. to de parejas de decisiones o, aún, una pareja de conjuntos de decisiones. La escala puede servir entonces para periodificar una crónica real, sin ninguna búsqueda de causalidad.

Esta aclvertencia teórica tiene una gran importancia si se consideran los grados del 1 al 44 (el primero y el último): l: crisis ostensible; 44: guerra de "espasmo". o guerra insensata. Ambos son difíciles de definir con el criterio unilateral/bilateral que se ha clido aqui: en efecto, en el primer grado, ¿cuál es la palabra que abre el escalón?, ¿es la palabra "crisis" (bilateral) o la palabra ostensible (unilateral)?

El grado 44 es, a la vez, un foso que sirve de limite a todos los escalones y a cada escalón, y una decisión tomada por un lado, o por ambos lados, conduciría a decisiones más allá de las cuales son concebibles otras decisiones en el caos que sigue al mayor intercambio nuclear. En los dos extremos de la escala hay una ambigüedad; en la misma escala hay una mezcla, por ejemplo, el $18^{\circ}$ escalón se llama "demostración espectacular de la fuerza"; y el gn: "confrontaciones militares dramáticas".

¿En qué puede ayudar estc breve análisis a nuestro tema? Primero que nada hay que advertir que el profesor $H$. Kahn no es representante oficial del pensamiento de los Estados Unidos. Sin embargo, no es necesario insistir sobre la influencia que han ejercido sus escritos ni, sobre todo, acerca de su carácter sintomático en cuanto a la formación de cierto tipo de reflexión estratégico-política norteamericana. Digamos que, en razón del poderíó global de los Estados Unidos y de la extensión cualitativa y cuantitativa cle sus medios de acción, no parece esencial a una política general de disuasión entrar en el detalle de la decisión inicial que desencadena una crisis. Con los conceptos enunciados sólo podemos elaborar una reflexión sobre la buena gestión de un estado de crisis del sistema mundial, dada la oposicion de intereses entre los dos Grandes. Esto implica, de partida, una cierta indiferencia por:

1) Los intereses marginales, $y$

2) Los centros de decisión marginales.

Ahora bien, la evolución de Europa se orienta precisamente hacia el aumento de la autonomía de los centros de clecisiones marginales, vale decir, las pequeñas y medianas potencias. En todo caso, en el dominio político, que es aquel en el que se preparan las crisis. 
La teoría de la escalada ha șido creada para el caso de una gran oposición bipolar. No proporciona, en todo caso, ningún instrumento de reflexión o previsión para la gestión de los conflictos y crisis posibles en Europa, los que son de naturaleza esencialmente politica, ja que en Europa no puede aceptarse ni la más mínima guerra convencional. En su fundamento lógico, tampoco es apta para actuar sobre las causas del conflicto a fin de resolverlo. El caso de Vietnam cs una ilustración palpable del fracaso de la teoría de la escalacla como manera de actuar frente a una crisis concreta.

Más aún, la misma metáfora de la escalada es parte importante del extraordinario error de diagnóstico político sobre el que reposa toda la lógica norteamericana acerca de la crisis del Vietnam, al menos según las explicaciones que se han dado al pueblo norteamericano. El origen del conflicto es atribuido a la intervención norvietnanita, lo que se contradice con los hechos, pero se conforma a la imagen que el primer escalón ha de introducir necesariamente en st rersión unilateral para lanzar la escalada como reacción norteamericana. Pero después de 1965 el aumento estadístico prosiguió de una manera preventiva, sin que se turiera en consideración la sabiduria de un viejo proverbio de Lao-Tsé, fundador del taoísmo: "cuantos más soldados haya, más bandidos habrá".

La aplicación de la gradación de escalada a escenarios reales implica la búsqueda ambigua de un enemigo que simboliza, no una decisión, sino lá coyuntura desfavorable, y una coyuntura que simboliza la superioridad de los Estados Unidos.

Contrariamente a lo que.se ha pensado al respecto, la aplicación de la escalada no puede "llevar los mismos mensajes" que la diplomacia, aun la más amenazante, y si prepara una negociación, es de la misma manera como cualquier otra guerra lo ha hecho.

\section{Dominación Cuantitativa}

Esta ojeada somera sobre algunos términos claves del nuevo vocabulario estratégico sería incompleta si no se diera cuenta de uno de los objetivos perseguidos en esta búsqueda del equilibrio EsteOeste: esta voluntad de dominar los armamentos no descubría el verdadero objetivo de la administración, que no consistía, de ninguna manera, en llegar a la paridad, ya que, como lo hemos visto, el missile gap no ha existido nunca, sino de aventajar netamente a los soviéticos en la carrera armamentista, obligar a la Unión Soviética a ahogarse económicamente en esta carrera y persuadirla de que renunciara a ella. En 1963 se esperaba que los Estaclos Uniclos, gracias a la clara superioridad cuantitativa que tendrian sobre los. 
soviéticos, podrian pretender conducir una estrategia antifuerza, cs decir, serian teóricamente capaces de destruir las fuerzas del adversurio y conservar una capacidad residual anticiudad destinada a disuadir al adversario de decidirse a ejercer. ụna represalia sobre las ciudades norteamericanas. Según la opinión corriente en ese tiempo, la estrategia antifuerza no podría mantenerse sino entre $1966 \mathrm{y}$ 1970, aproximadamente.

Numerosos expertos han considerado esta idea como la demostración de una estrategia operacional agresiva (la noción de "primer golpe" sería sinónimo de "agresión") o como un mito tecnológico desprovisto de realidad; y hasta como una imposibilidad material.

Sin embargo, hay que advertir que, aún hoy dia, existe en Estados Unidos la tendencia a admitir que los soviéticos han sido derrotaclos en la carrera armamentista y que aceptan esta derrota. Lo que explicaria, en gran parte, su prudencia actual.

Esta descripción me parece inexacta, porque descansa sobre la idea de que, en un momento dado, los soviéticos no han sido prudentes y oculta el hecho de que, partiendo de nada, han avanzado cada vez mús hacia el equilibrio. Esto es hacer poco caso a toda la historia cle la guerra fría y comenzar la reflexión política al día siguiente de la crisis cubana, que fue el resultado de lo que una buena parte de la opinión mundial considera, en efecto, una imprudencia soriética (en especial los norteamericanos y los chinos).

Empero, los Estados Unidos poseen una considerable superioridad cuantitativa en vehículos, lo que fue valorizado en la estrategin de MicNamara, y en la que descansa, en gran parte, el sentimiento de seguridad de los Estados Unidos. Para citar algunas cifras, recordemos que, a comienzos de 1964, el Oeste disponía de 475 rCBAr, mientras el Este poseía solamente 100. De 1964 hasta los comienzos de 19.67, estas cifras serán, respectivamente, de 934 para el Oeste y 300 para el Este, según estimaciones del Institute of Strategic Studies de Londres. En el campo de los cohetes de capacidad intermedia sobre submarinos de tipo Polaris, se pasa, entre las mismas fechas, de 192 cohetes occidentales contra 100 cohetes orientales a 624 cohetes occidentales contra 150 orientales. Advirtamos que si los Estados Unidos aventajan ampliamente a la Unión Soviética en el ritmo de la carrera por los cohetes en submarinos, con una tasa de crecimiento global de $320 \%$ contra $150 \%$ solamente para la Unión Soviética entre comienzos de 1964 y comienzos de 1967, no sucede lo mismo con la carrera por los cohetes intercontincntales, ya que la tasa de crecimiento correspondiente es de $190 \%$ en los Estados Unidos con- 
Alain Joxs / Hacia una nueva Concepción de la Seguridad Europea

tra $300 \%$ en la Unión Soviética, la que si bien se mantiene todarfa en inferioridad numérica, asciende bastante rápido.

Por lo demás, esta comparación casi carece de sentido si no se tiene en cuenta la profunda diferencia de estrategias y el poderío de las cabezas nucleares.

Además la-Unión Soviética está decidida a proseguir, a paso lento, la construcción de un sistema de cohetes anticohetes alrededor de algunas de sus ciudades, dejando entender que se trata de precaverse contra la China. Este dispositivo, que equivale a una "capacidad antifuerza de segundo golpe", por así decir, tendría el inconveniente, según los Estados Unidos, de perjudicar el equilibrio actual. La carrera no está, pues, terminada aún y puede dar lugar a nuevos incidentes en el recorrido.

Sin embargo, el retorno a un gran desequilibrio nuclear, provocado por un "despegue tecnológico" que podria llevar a uno u otro de los adyersarios a una guerra nuclear preventiva o a una agresión, se ve poco probable. El equilibrio nuclear parece haber sido logrado por mucho tiempo. Tanto la Unión Soviética como los Estados Unidos pueden tener el propósito de sofocar económicamente al adversario más que de obtener una superioridad aplastante sobre él.

No obstante, una de las finalidades de la superioridad cuantitativa que se perseguia con la producción en masa de Minulcman y Polaris fue de poder, en el caso específico en que estuviera en juego el interés de los Estados Unidos, no solamente disuadir al adversario, smo además disuadirlo de reacçionar ante una intervención directa o indirecta de los Estados Unidos en su propio espacio, y ganar una guerra limitada, no nuclear, a cubierto de la disuasión nuclear superior de la que se dispondría. El aspecto "antifuerza" global de la doctrina McNamara ha desaparecido rápidamente, pero lo que no ha desaparecido es el espíritu contraofensivo inspirado por la conciencia de cierto tipo de superioridad norteamericana en el dominio de la abundancia nuclear y de la sofisticación de la reflexión estratégica. Por otra parte, la crisis cubana de 1962 sirve también como ejemplo a la escuela que pretende que, en una operación local, lo que importa es la superioridad clásica, reservándose a la superioridad nuclear la misión de evitar el chantaje nuclear. Por lo demás, los Estados Unidos se preparan para poner en práctica, desde 1970, el sistema de intervención clásica rápido y extraordinariamente poderoso y eficaz.

II

\section{LA Situactón Agtual}

Entramos ahora en nucstra. segunda parte: la situación actual er Europa. 
En el Ocste, los Estados Unidos han desarrollado, hasta sus últimas c.nnscumencias, sus teorías sobre la guerra controlada, la decisión controlada, la respuesta flexible y los dispositivos de planificación del comando y del control de las decisiones necesarias. Pero este conjunto teórico y práctico no podría servir a una política norteamericana en Europa sino a condición de que se hubieran realizado dos hipótesis sobre la evolución clel mundo:

1) Que el gran propósito de Kennedy de formar una Alianza Atlántica más coherente, tanto política como militarmente, hubiera dado resultado. Ahora bien, la estrategia norteamericana no ha sido aceptada oficialmente por la orAN antes de este año (es decir sin la Francia), y presenciamos el hundimiento del gran propósito, en el cual no se escogió con claridad entre reforzar el leadership norteamericano o formar un partnership con una Europa consolidada.

21) Que el mündo hubiera continuado siendo bipolar; ya que el conflicto chino-soriético modifica las condiciones de una gran negociación permanente entre el Este y el Oeste.

En estas condiciones, era inevitable cierta desconfianza de parte de los europeos, aunque la política norteamericana inaugurada por la administración Kennedy se hubiera dirigido enteraménte hacia esta confrontación razonable, y no, como lo creyeron algunos, hacia una mezcla inquietante de desprendimiento marginal y de aventura, consecuencia de la conjunción entre flexibilidad y dominio en armamentos. Esta desconfianza da lugar al renacimiento de cierto neonacionalismo, que es en verdad el renacimiento de una imaginación política local alienada desde hacía mucho tiempo.

En el Este, la Unión Soviética, al alejarse francamente de China, no se ha propuesto, ciertamente, debilitar el bloque socialista, sino más bien reforzarlo alrededor del Pacto de Varsovia, para manejar, en un campo más reducido, pero más homogéneo, una negociación general con los Estados Unidos. Lo que los Estados Unidos trataban de obtener por la presión económica y el rodeo de una doctrina militar de integración, la Unión Soviética pretendía lograrlo por el reagrupamiento ideológico $y$ el partnership político y militar. Ahora bien, en este campo los particularismos nacionales tienden también a afirmarse cacla vez más cómodamente. La actitud particular de Rumania, por ejemplo, y la resonancia poco visible, pero no menos cierta, que su actitud tiene, por lo menos en los países balcánicos, acarrea dificultacles en la coordinación militar. Estas dificultades son, en realidad, tolerables para la Unión Sorićtica, mientras no haya rics... de guem, prom no hon sirh, ericlentemente, deseadas. 
Asi vemos como el desmantelamiento de las dos alianzas prosigue, sin poder alcanzar un punto de equilibrio, ya que no se produce paralelamente y está fuera del control total del líder.

En estas circunstancias, ¿qué sucede con la seguridad europea?, cs decir, con la seguridad de los regímenes de los países de Europa y al sentimiento de seguridad de los pueblos frente a las amenazas de dominación exterior.

¿Cómo puede garantizarse esta seguridad, ahora y en el porvenir, puesto que ha escapado al control de las dos alianzas?

Haremos un intento por describir la composición actual de la seguridad europea descle dos puntos de vista. Primeramente, describiendo el papel que las Europas continúan desempeñando dentro de los sistemas instaurados por los bloques. Y, por otra parte, describiendo el estado de la negociación multiforme sobre lo que se ha llamado la reunificación de Europa.

En lo que respecta al papel estratégico desempeñaclo por Europa en la Alianza Atlántica, recordemos rápidamente las etapas precedentes:

Desde la fundación de la OTAN hasta 1954, el papel que correspondía a Europa era, claramente, asegurar una defensa clásica por medio de un combate destinado a ganar tiempo sobre el Rhin y proteger las bases del SAC. Los ejércitos eran el escudo y el sAC la espada.

Más tarde, xon la aparición de las primeras armas nucleares soviéticas, se abandonó progresivamente la idea de un combate para ganar tiempo. Este plan ya no tenía sentido en un ambiente nuclear y los alemanes, que hacian sir su voz nuevamente, deseaban que el combate fuera llevado lo más adelante posible para evitar la invasión completa de la República Federal Alemana en las prímeras horas de la guerra.

Hacia 1954, se pensaba que los europeos debian asegurar una defensa en profundidad, combinada con una poderosa acción del SAC desde el comienzo de las hostilidades, asegurándose un plazo de algunos días para el despliegue avanzaclo de una cortina defensiva liviana y mụy móvil, equipacla con armas nucleares. tácticas. Los efectivos considerados eran poco numerosos (200.000 hombres).

Esta doctrina defensiva ha sido adoptada por los gobiernos y constituye, en principio, la estrategia de la oran hasta nuestros días. Pero, a partir de los años de transición 1957-1960, y sobre todo del advenimiento del Presidente Kennedy, sé hizo evidente que los aliados ya no podían contar con una respuesta automática; la única capaz, según ellos, de disuadir al adversario de emprender acciones limitadas sobre el territorio europeo. Comienza a comprenderse que el in- 
terés de los Estados Unidos puede ser distinto al de Europa Occidental.

Para evitar el desarrollo de estas ideas, los Estados Unidos recurrieron a una serie de expedientes:

1. El encargo de conventional build-up, después de la reevaluación de los efectivos soviéticos. La fuerza nuclear pasaba a ser el escudo y la fuerza clásica la espada. Se planteó la cuestión de saber por qué la guerra clásica volvía a ser posible siendo que se había demostrado la incompatibilidad en un mismo escenario entre la utilización de fuerzas clásicas y de fuerzas nucleares, ya que las primeras exigen la concentración de tropas y las segundas, por el contrario, su dispersión.

2. La pausa: En caso de comenzar un ataque soviético, los Estados Unidos se reservaban una pausa, en el curso de la cual hacian llegar un mensaje; esto podía significar que los soviéticos se habrían sentido libres de ocupar una parte de Europa, disponiéndose a negociar después.

3. La MLF (Mulilateral Force) para evitar tomar" en considera(ión el despliegue de arkix (Medium Range Ballistic Missiles) propuesto por Norstad, antes de su dimisión.

\section{El Targetting común (comité MCNamara).}

5. Por último, el considerable aumento de las dotaciones en armamento atómico de las grandes unidades norteamericanas de Europa $y$ el incremento notable de cabezas nucleares, almacenadas en el -continente sobre todo para manifestar la importancia que los Estados Uniclos atribuían a Europa. El aumento de los dispositivos nucleares en Europa fue realmente considerable.

En el nivel cle la división, el número de los dispositivos de lanzamicnto nuclear pasó de 8, en 1964 (cañón de 203 y rampas de Fonest Johm), a 28 para las clivisiones de infantería y 80 para las divisiones blindadas, en 1956, gracias a la puesta en servicio de los cohetes Pcrshing (primer modelo de $400 \mathrm{Km}$. de alcance), de los cohetes Sergrant, a la nuclearización de la arilleria de 155 , etc. Tanto, que el número total de cabezas nucleares dispuestas en Europa habría pasado de 3.000 en 1961 a 7.000 en 1966. El grueso del aumento se ha hr. ho en los últimos tres años. 
Actualmente, debido: 1 ?) al retiro de Francia de la otan, lo que disminuye el espacio y plantea problemas logisticos, y 2\%) al poco éxito de su petición de aumentar los medios militares clásicos europeos, los Estados Unidos están quizás orientándose nuevamente hacia la idea de la respuesta inmediata y el despliegue de Mrbm, lo que constituye indudablemente un triunfo para las tesis francesas y alemanas, pero, a la vez, una prueba del extremo empirismo de las teorías norteamericanas.

Debemos preguntarnos, entonces, cuáles son los factores profundos de estos cambios ondulantes de la estrategia norteamericana.

Examinaremos primeramente los factores financieros: las dificultades del dólar tienen repercusión sobre los dispositivos militares. Recordemos que la balanza de pagos de Estados Unidos está constantemente en déficit y que los stocks de oro norteamericanos bajan regularmente desde 1949. Los déficit más importantes fueron los de 1959 y 1960 . (3.700 y 3.900 millones de dólares, respectivamente) y la balanza de pagos había mejorado desde 1961: el déficit ascendió entonces solamente a I.300 millones de dólares en 1965, pero será sin duda de dos mil millones en 1966.

Uno de los mejores recursos para paliar este déficit es la venta de armamentos (que desde 1963 ha reemplazado completamente a las entregas gratuitas), y particularmente, la venta de armamentos a los países industríalizados de Europa.

Europa Occidental conserva, pues, un papel determinante en el mantenimiento de la seguridad occidental en general, pero los aspectos militares de su unión con los Estados Unidos son menos importantes que los aspectos económicos y financieros, aun en el dominio de los armamentos. Algunas personas llegan hastá a decir que la OTAN se ha transformado en una agencia de venta de armas norteamericanas más que en un sistema de seguridad colectiva. En este respecto, Alemania Occidental constituye lo que podríamos llamar la gallina de los huevos de oro de la otan.

Por una especie de chantaje a la seguridad, la presión norteamericana ha tenido siempre éxito en este país, ya que es el único en Europa Occidental donde el temor a una invasión soviética se mantiene vivo, por razones fáciles de comprender. Sin embargo, parece que los Estados Unidos se han portado recientemente demasiado insistentes, en un momento en que, por lo demás, las circunstancias financieras no eran fayorables. La presión sobre Alemania para obtener el aumento de los gastos de estacionamiento de las tropas norteamericanas sobre el territorio federal, parece haber fracasado. La misma suerte ha corrido la insistencia con la que ofrecen a los alemanes cohetes 
Pershing en reemplazo de los Starfighters desnuclearizados (inconvertibles en aviones de "interdicción" clásica después de las transformaciones de que habian sido objeto).

Digamos al pasar que el cohete Pershing es una cosa seductora desde el punto de vista técnico, pero tal vez peligrosa desde el punto de vista político. En su última versión, este aparato, concebido para transportar una cabeza nuclear y que está dispuesto sobre rampas muy móviles (se desplazan a la velocidad de un tanque), provisto de un aparato electrónico de puntería y de comunicación, posee un dcance de 1.000 a $1.500 \mathrm{Km}$, según las fuentes. Se trata, por to tanto, de un MRBM móvil, capaz de golpear todo el territorio polaco y tal vez una parte de Rusia. Parece que se vuelve a la solución propuesta por el general Norstad antes de su dimisión. Pero los tiempos han cambiado y el ofrecimiento no se ha hecho sino a los alemanes. Estos, fuera del precio por pagar, se sienten quizás poco inclinados a hacer frente solos a delicados problemas de targetting.

En estas circunstancias, la balanza militar de pagos al extranjero se cierra nuevamente con un déficit igual al doble de las entradas. Para Europa, incluyendo a España, el costo de la presencia militar norteamericana asciende a 925 millones de dólares.

Por otra parte, la guerra del Vietnam acarrea gastos ćada vez más pesados. En el presupuesto militar de $1966-6 \%$, que representa el $53 \%$ dêl presupuesto federal, la parte correspondiente a la guerra del Vietnam representa un 20\%. El costo global de 1965-66 era de 4:600 millones de dólares. Para 1966-67 ya se ha calculado en 12 mil millones de dólares; tales son los aspectos financieros.

Respecto a los factores militares, recordemos que alrededor del $25 \%$ de las fuerzas arrnadas norteamericanas están ocupadas o directamente relacionadas con la guerra del Vietnam. $30 \%$ de los efectivos del ejército de tierra-y de los marines están Iuchando alli, pero, en realidad, entre 7.5 y $80 \%$ de los efectivos están, de cerca o. de lejos, relacionados con esta guerra o con $s u$ preparación.

- En lo que toca a la aviación, el $75 \%$ de la aviación de transporte está orientada hacia el Pacífico.

Este esfuerzo no hace sino perturbar las otras tareas que se ha propuesto el potencial militar norteamericano.

Este conjunto de factores: financieros, militares y políticos, parece llevar a Estados Unidos a aligerar su aparato en 'Europa.

Esta tendencia se manifiesta en los diferentes niveles de la opinión autorizada en los Estados Unidos, incluyendo la de los más responsables: pudimos notar las declaraciones hechas en el Senado por el senador Mansfield, el 27 de julio del año pasado; varias tomas de po- 
sición de senadores demócratas; y, por fin, la entrega, el 31 de agosto


tendiente a reclamar una "reducción substancial cle las tropas norteamericanas en Europa". A pesar de los desmentidos del Ejecutivo, parece que existe un acuerdo tácito con la Unión Soriética, bastante avanzado en este sentido. Pero, en el estado actual de la guerra de Vietnam, es difícil que los dos Grandes lleguen a concluir un acuerdo formal sobre Europa. Podríamos decir que la estabilidad política en Europa es lo que permite, en gran parte, el esfuerzo de guerra de los Estados Unidos, pero esto no garantiza su defensa; al contrario, la misma Europa es quien asegura, políticamente, su seguridad.

En lo que concierne a los países secunclarios clel Pacto de Varsovia, la historia de su actuación está en cierta forma desconectada de la gran problemática que hemos descrito al trazar las etapas de la confrontación entre el Este y el Oeste. Por razones geoestratégicas evidentes, nunca nos ha preocupado preguntarnos si los países de Europa Oriental son o no útiles a la Unión 'Soviética para resistir un ataque contra el sistema socialista o contra ella misma.

Los países socialistas de Europa han seguido, sin hacer ruido, la línea soviética clurante la mayor parte de la historia de la postguerra, formanclo parte de un teatro de opcraciones eventual inseparable de la Unión Sorićtica. Sin cmbargo, es intcresante seguir su evolución, que hoy día se ha ruelto singular en ciertos aspectos, ya que, precisamente a causa de esta evolución, podremos hablar cle un sistema de seguriclad europea que no sea simplemente el resultaclo de un acuerdo entre la Unión Soviética y los Estados Uniclos, o, lo que sería aún más incompleto, de una negociación entre Francia y la Unión Soviética.

Al nacer, en 1955, el Pacto apareció como una respuesta a la inclusión de Alemania Occidental en la OTAN, más que como un medio serio de integración militar. Las ínicas fuerzas militares que realmente cumplian eran las fuerzas sorićticas $y$ existian acuerdos bilaterales entre cada país $y$ la Unión Soviética, que manifestaban el predominio sovietico. Después del asunto de Hungría, en 1956, la Unión Soriética se ha visto, probablemente, obligada a crear una organiza-

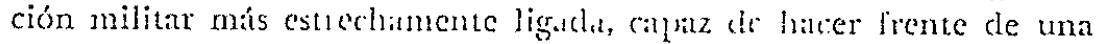
manera colectiva at la eventualiclad de una "nueva Hungría". Al menos, esta es una explicación norteamcricana que parece estar intimamente vinculada a la idea cle "fuerza de intervenciún panamericana". Es probable que la efectiva liberalización que sucedió al asunto húngaro haya hecho deseable y posible la modernización de los ejércitos de Europa Oriental y su fuerte integración en uma organización mili. 
tar común, dominada por la Unión Soviética, pero en la cual hasta entonces ésta no se había interesado mayormente.

Las primeras maniobras de conjunto de los ejércitos del Pacto de Varsovia tuvieron lugar en octubre de 1961, con la participación de la Unión Soviética, Polonia, Checosloraquia y Alemania Oriental. Tuvieron particular importancia "para la elaboración de miras comunes sobre los métodos de guerra nuclear y convencional", pero, hasta los alrededores del verano de 1964, los ejércitos satélites estaban organizados y entrenádos solamente para la guerra clásica. Sus efectivos hân permanecido extremadamente estables, con 900.000. a 1.000.000 de hombres solamente, repartidos de la siguiente manera:

Polonia, con 50 divisiones y 1.000 aviones; Checoslovaquia, con 40 divisiones y el segundo lugar, después de Polonia, en aviación. Alemania del- Este posee efectivos más reducidos, pero dotados de equipos puestos al día por la entrega de armas soviéticas del último modelo. Las tropas soriéticas tenían veinte divisiones en Alemania Oriental, dos divisiones en Polonia, cuatro en Hungria, y unidades de "misiles tácticos" con capacidad nuclear y clásica.

Hacia el yerano de 1964, la diferencia entre los ejércitos soviéticos nuclearizaclos y los 'ejércitos satélitos clásicos comienza a atenuarse.

Los soviéticos distribuyen a los ejércitos de Europa Oriental sistemas de vehículos provistos de una capacidad nuclear en cohetes tácticos de 150 millas de alcance, cuyas cabezas son colocadas bajo control soviético. Estạ etapa nuclear sharing continúa después de la partida de Khruschev (fines de 1964).

El conjunto de la política en 1960 se puede explicar de diversas maneras: política de economia presupuestaria por reducción masiva de los efectivos del ejército e insistencia en la capacidad de disuasión de las nuevas Fuerzas de Cohetes Estratégicos constituidas en la Únión Soviética. En ese momento había que encontrar una compensación y' se atribuyó a los ejércitos satélites una parte de la carga convencional. Una consideración también de orden estratégico pesaba en la balanza cuando se afirmaba que en Europa cualquier conflicto se transformaría rápidamente en nuclear. En caso que se produjera un tiro de "interdicción" sobre las lineas de comunicación con la Unión Soviética, se hacia necesario que la fuerza local fuera capaz de resistir un ataque hipotético sin contar con la ayuda inmediata de los refuerzos soviéticos. Por último, era menester que el Pacto de Varsovia tuviera algún peso para que ofreciera un apoyo político durante la construcción del muro de Bexlín, la que fue decidida el 31 de agosto de 1961, al día siguiente de una cleclaración del Pacto de Varsovia. 
Estas explicaciones son de tipo unilateral: ellas explican lo que sucede en el Pacto de Varsovia por lo que sucede en el Pacto de Var. sovia. Hay otro tipo de explicación ciertamente más racional que consiste en explicar to que sucedió en el Pacto de Varsovia por lo que sucedía en el mismo tiempo en la OTAN.

Si nos transportamos a Jo que clecíamos más arriba sobre la evolución de la estrategia norteamericana, comprobaremos paralelismos sorprendentes en las medidas tomadas (nuclearización de las tropas norteamericanas, pero reticencia a nuclearizar las tropas aliadas; demanda de un aumento de la participación clásica; integración reforzada, a fin de conservar el dominio del aparato de decisión). Pero la cronología de estos diversos elementos no es la misma, y a la vez, las consecuencias o las implicaciones políticas no son tampoco las mismas, lo que se debe particularmente a la diferencia de las relaciones geoestratégicas entre aliados.

Esta manera de formular las cosas nos permite distinguir con bastante claridad los elementos de la evolución del problema de la seguridad europea que dependen estrechamente de la evolución de la tecnología de los armamentos, y aquellos que dependen, por el contrario, de las condiciones puramente locales y, por asi decir, contingentes, que son las condiciones geográficas e históricas del sentimiento de seguridad de los diferentes pueblos.

La mayor cohesión militar aparente del Pacto de Varsovia, en comparación con la Alianza Atlántica, se debe, sin duda, hoy día, a estos elementos accidentales (miedo del peligro alemán, continuidad terrestre de los países de la Alianza). El relativo debilitamiento de su. cohesión se debe, por el contrario, a los fenómenos de orden general que se sitúan también en el origen de la dislocación de la Alianza Atlántica (problema del reparto de responsabilidades de la decisión en una tecnología que impone decisiones en plazos muy breves); y también al puro y simple ejemplo de lo que sucede en el seno de la othN (el caso francés parece inspirador del caso rumano).

Hoy en clía, el asunto de una eventual división del control de las armas nucleares se ha hecho más insistente. En el curso de la séptima reunión del Comité Consultivo Político del Pacto de Varsovia una declaración soviética ha hecho alusión a la existencia de fuerzas nucleares conjuntas en el Pacto. Pero, en realidad, no se sabe nada de la manera cómo están organizados el comando y el control de las fuerzas nucleares dentro del Pacto. Por otra parte, existe una reivindicación general de los estados-miembros para tener mayor peso en la Alianza, a cambio de una integración más activa. Esta tendencia se ha acentuado después de la última reunión del Pacto, en Bucarest, 
b, como el. Occidtnte, liene por resultado la formación de dos tendencias: los polacos, los checos y los alemanes estín muy deseosos de mantener el Pacto y se conducen de manera similar a la República Federal. Los países balcánicos, menos preocupaclos por el problema alemán, tienen menos razones para interesarse en el Pacto. En Bulgaria ha habido también, al parecer, alguna agitación debido a la existencia de una fracción nacionalista dentro del ejército; mientras los rumanos empujan esta actitud hasta sus límites al manifestax casi públicamente que no quieren pagar mayores compromisos de la Alianza, cuya conveniencia no les parece clara.

De todas maneras, estos aspectos conjuntos de la evolución del Pacto de Varsovia: modernización, integración, aspiración a la división de las responsabilidades, demanda de autonomía de las decisiones en la Alianza, no pueden ser considerados simplemente como aspectos concomitantes. Hay una cronología, y el aspecto "refuerzo" se dio con anterioridad a las reivindicaciones de autonomía que ese refuerzo hizo posible. Es por eso que yo pienso que lo que actualmente informa esta evolución no es el refuerzo, tal como querrian hacerlo admitir los alarmistas y los partidarios de un retorno a la integración y al reagrupamiento en bloques, sino más bien la vuelta a cierta autonomía de juicio y de decisión en los paises de Europa Oriental. Esta autonomía de juicio está enteramente orientada hacia un arreglo pacífico y clefinitivo de los asuntos nacionales en Europa.

\section{Reunificación DE EUROPa}

Para concluir, esto nos conducirá a algunas consideraciones sobre el estado actual de las negociaciones de lo que se ha dado en llamar "la reunificación de Europa", y sobre la lógica de esta negociación o, más bien, las diferentes condiciones lógicas de las diferentes negociaciones ocultas bajo esta expresión.

Ante todo, haremos algunas consideraciones doctrinarias sobre el teatro de negociaciones europeo. El concepto de coexistencia pacífica se ha desarrollado en el Este, despućs de la muerte de Stalin, para tener en cuenta, aunque teóricamente, el fracaso de las previsiones sobre la gran crisis general del capitalismo.

1. El análisis soviético reposa sobre la comprobación dẹ que es la economia de guerra lo que permite a los Estados Unidos enjugar la inevitable superproducción y evitar el desequilibrio entre el crecimiento de los medios de producción y el crecimiento de los mercados de consumo. 
En esta perspectiva, el aflojamiento de la tensión podría ser favorable al campo socialista, ya que debería conducir a la eliminación de la economía de guerra $y$, por lo tanto, a crisis más marcadas en el munclo capitalista, y especialmente en Estados Unidos:

2. A esta interpretación se opone otra idea, según la cual, puesto que el neocolonialismo procura una prórroga a la muerte del capitalismo, el movimiento revolucionario debería orientarse hacia el mundo subdesarrollado y ayudar a los movimientos de liberación nacional. Esta tesis aparece en Más Vale Menos Que Más, publicado por Lenin en 1923, y es una de las bases de la estrategia preconizada por la China.

Según la tesis china, la coexistencia no debe constituir la línea general del movimiento revolucionario sino solamente una línea particular concerniente a la "segunda zona intermediaria", es decir, los países occidentales y el Japón, más algunos estados asiáticos vecinos y neutros (Pakistán, Birmania, Cambodia), pero de ninguna manera los Estados Unidos, cabeza del "imperialismo mundial". Así, el gobierno de la Unión Soriética, por razones de orden teórico general y por razones empiricas locales, descarta actualmente -en un debate político, abierto y público- toda posibilidad de guerra en Europa. Por otro lado, la China, junto con empujar a la Unión Soviética a abrir un segundo frente en Europa destinado a aliviar al Viemam, Ina teorizado también sobre la coexistencia pacífica con Europa. Se mantiene, por lo tanto, en cierta contrádicción, de la que no puede salir dada su incapacidad de actuar realmente en este frente.

De lo dicho resulta, según mi parecer, que toda acción soviética general en contra de la Europa Occidental debería estar precedida de un debate de reconsideración de ideas y de un acercamiento a Chịa; lo que parece imposible dada la firme resolución de la política soviética. De todas maneras, Europa cuenta con un plazo de alerta que no tiene nada que ver con los plazos de alerta de algunos minutos que se conocen en la estrategia nuclear y que, por una aberración que yo llamaría militarișta, son los únicos que se consideran como fundamento de la estrategia de una nación.

Sin embargo, esta interpretación es discutible y actualmente se discute en verdad; recordemos to que decía el Secretario General de la ótAN, el señor Manlio Brosio, con motivo de la última reunión de esta organización, êl 14 de noviembre, en París. Desarrolla las siguientescideas 
19 Es falsa la idea de que el bloque socialista se desintegra, ya que existe una cohesión perfecta entre los paises satélites y la Unión Soviética, tanto en los asuntos militares como en los politicos, y con Ia China no hubo nunca cohesión en el período anterior.

22 La idea de que la Unión Soviëtica ya no es una potencia agresiva hace caso omiso de una reflexión estratégica que debe considerar lo posible, $y$ provicne en parte de la idea de que el bloque se desintegra.

39 El cisma chino no ha teniclo repercusión sobre la liberalización de los satélites, la cual, por el contrario, es el resultado de la presión ejercida por la unidad occidental.

Esta descripción puede considerarse como un último esfuerzo por enfocar los asuntos europeos con un criterio regional, ya que se describe el sistema europeo como un sistema en sí, y no como una parte del sistema Este-Oeste o, mejor dicho, del sistema mundial. Este intento se contradice con el enorme esfuerzo educativo hecho por los Estados Unidos para hacer comprender a los responsables o a los especialistas europeos, los caminos y los medios de la nueva estrategia norteamericana, la cual descansa en una visión realmente mundial de la oposición y en una concepción central de la guerra y de la disuasión.

Como, además, no toma en cuenta la realidad política local que se supone ella debe influenciar, podemos afirmar que se trata de una descripción realmente superada.

Otra visión política. se refleja en un artículo muy interesante, American Policy and the New Europe, aparecido en el Foreign Affairs. de octubre de 1966. El autor, el señor Frank Church, hace una crítica a los comentarios virulentos contra la política francesa del señor McGeorge Bundy, y afirma que la politica del General De Gaulle no está aislada en Europa, adliniéndose a la idea sostenida por el profesor Kissinger, según la cual la reaparición del nacionalismo europeo, es decir, el mantenimiento del pluralismo, no constituye un perjuicio para la política norteamericana, ya que no es probable que la paz mundial esté mejor asegurada con la aparición de un estado europeo del tamaño y la potencia de Estados Unidos o la Unión Soviética.

El autor critica la decisión que parece haber tomado el Departamento de Estado, según la cual Washington preferiria ligar a Alemania más estrechamente a la oTas desmembrada, con la esperanza de 
un acceso indirecto al armamento nuclear, que mejorar las relaciones entre los Estados Unidos y Rusia. Según él, dada la despolarización actual, los Estados Unidos deberían estar a la cabeza de sus aliaclos europeos en el "moviniento hacia el Este", sin lo cual los europeos de todas maneras continuarán en esta dirección, volviendo la espalda a los Estados Unidos, si es necesario.

La política de los estados europeos, considerados individualmente o en conjunto, no podrá ceñirse, indudablemente, a ninguna de estas dos tesis:

Elegir el pluralismo, permitiendio asi a los Estados Únidos ponerse a la cabeza de la negociación con el Este, no puede ser un objetivo europeo, aun cuando, ineluctablemente, éste será el resultado de la dispersión de las iniciativas políticas $y$, sobre todo, de la falta de coordinación entre la política francesa y la alemana. Por otra parte, cl rígido mantenimiento de la Organización del Atlántico Norte y cle las categorías de peligro y seguridad, propias de la época de la guerra fría, no puede resistir por más tiempo al flujo de la opinión pública.

El porvenir debe traer, más bien, un estrechamiento de la cooperación y un aumento de los intercambios diplomáticos entre los estados europeos de ambos lados de la cortina de hierro, de manera que la negociación no sea llevada adelante por la sola iniciativa de los Estados Unidos. Ia idea más sencilla es que una negeciación conducicla y aceptada por los europeos no puede ser maja para los Estados Unidos, en tanto la proposición inversa puede ser falsa.

Actualmente esta negociación se sitúa en diferentes niveles, los que sería útil recordar para poner un poco de orden en esta problemática. Cada tramo de negociación tiene su propia lógica y es capaz de tratar solamente algunos problemas.

Estos niveles son los siguientes: el nivel Washington-Moscú; el nivel Alianza Atlántica-Pacto de Varsoria; el nivel de relaciones bilaterales intraeuropeas -en un caso particular: las relaciones entre Alemania Oriental y la Alemania Federal.

Entrelazadas a estos conjuntos están las relaciones que los paises europeos medianos o pequeños tienen con sus respectivos conductores.

El nivel Washinglon-Moscú dio lugar a ciertas negociaciones que tuvieron éxito al dia siguiente del asunto cubano, pero aparte del asunto cubano mismo: el acuerdo sobre el teléfono rojo del 20 de junı de 1963, y el trataclo de prohibición de los ensayos nucleares, a excepción de los ensayos subterráneos (5 de agosto de 19:53). El perfeccionamiento de este tratado hasta llegar a comprender todos los 
ensayos subterráneos, prosigue con buenas perspectivas de éxito, debido a los progresos registrados en la detección y el análisis cle los sismogramas, y a la voluntad política de ambos lados. Estas negociaciones no interesan sino indirectamente a la seguridad europea, a través del caso alemún. Lo mismo sucede con las negociaciones sobre la no proliferación, tratada en el marco de la Conferencia de los Dieciocho, en Ginebra.

Por el contrario, una negociación Washington-Noscú que diera por resultado la disminución de las tropas soviéticas y norteamericanas en Europa, sería de una gran importancia. Sin embargo, en el estado actual de las cosas, ambos países parecen incapaces de conseguir algo más que una disminución conjunta, sin gran ruido, en caso de que se lo propongan; pero esta modificación no llevaría de ninguna manera a una negociación política: la guerra del Vietnam los obliga a no entenderse demasiado abiertamente $y$, por lo demás, los países europeos, en particular las dos Alemanias, no están dispuestos a sentarse a una misma mesa de conferencias para tratar de problemas que replantearian la confrontación en Europa. Dada la velocidad de los acontecimientos en Europa, podemos decir que el tramo WashingtonMoscú es capaz de mejorar las condiciones generales del aflojamiento de la tensión, pero es incapaz, hoy en día, de tratar los problemas políticos a la escala europea.

El nivel Pacto Atlántico-Pacto de Varsovia tiens, entre otros, el mérito de englobar a los países europeos directamente interesados y también de reunir dos entidacles que se niegan recíprocamente los fundamentos de su legitimidad: la Alemania Oriental y' la República Federal Alemana.

En esté nivel se manifestaron algunos de los grandes propósitos de crear una zona tope en Europa Central; los cuales permanecen a la orden del día.

Recordemos que el proyecto de Eden de llegar a un acuerdo entre los Cuatro Grandes con una Alemania reunificada sobre una limitación de las tropas alemanas y la creación de una zona desmilitarizada entre el Este y el Oeste, fue expuesto en una época - 1955-en que el proceso del rearme alemán aún no se había concretado. El plan Kennan y la primera versión del plan Rapacki datan del año 1957. El primero preveía la evacuación simultánea de Alemania por los Estados Unidos y la Unión Soviética y el mantenimiento de las dos Alianzas. La Alemania reunificada debería poder elezir libremente su destino: el Este, el Oeste o.la neutralidad. El plan Rapacki preveía la desnuclearización de una zona que comprendía las dos Alemanias, 
Alain Joxe / Hacia una nueva Concepción de la Seguridad Europea

Polonia y Checoslovaquia. 1957 es también el año del Sputnik y el año en que se colocan armamentos nucleares tácticos en Europa.

Estos dos ejemplos nos demuestran que todas las proposiciones hechas a este nivel de reflexión y de negociación estaban - tanto por sus contextos como por la intención de sus autores- guiadas por consideraciones militäres. Fueron también rechazadas por razones militares: el plan Rapacki, porque la pérdida de espacio occidental implicada en la desnuclearización de' Alemania no estaba equilibrada por desnuclearizaciones orientales, dada la extremada desimetria de los espacios disponibles por las dos Alianzas.

Estos y otros ejemplos mostrarian también que toda proposición de acuerdo sobre una zona limitada que comprenda Alemania se estrella contra el problema alemán. Ahora bien, el nivel interalianzas es preferido, en principio, por los polacos y los checos, por ciertos paises cle la OTAN y por las dos Alemanias, justamente porque parece garantizarlos contra ciertos trastornos muy peligrosos, sobre todo en Alemania.

Podríamos concluir que esta garantía contra el cambio, significa hoy día incapacidad para negociar cualquier cambio. La elección de dos pactos militares antagónicos para negociar o garantizar delicados asuntos de política y de cooperación, no es ciertamente lo mejor, sin contar con que sería necesario que estos pactos estuvieran habilitados para negociar mediante una delegación de soberania difícil de imaginar.

Faltando esta delegación de soberanía, el procedimiento por pactos no permite eludir el problema de la participación de los dos estados alemanes, o sea, no puede llegar a nada mejor que a un pacto formal de no agresión sin novedad política en su contenido. Un pacto de no agresión entre la oTAN y el Pacto de Varsovia podría tener tal vez un efecto psicológico favorable que ayudaría a aflojar la tensión, pero sería una negociación vacia como aquellas a las que nos ha acostumbrado el nivel Washington-Moscú.

Las relaciones entre las dos partes de Alemania son, evidentemente, muy importantes. Es la ausencia de relaciones lo que permite decir que las dos Alemanias están en una guerra civil en suspenso, y esta guerra civil en suspenso constituye hoy día el verdadero problema de la seguridad europea, junto al asunto de las fronteras occidentales de Polonia; que le estál ligado.

Evidentemente, el régimen de gobierno de Alemania Oriental no proviene de elecciones libres $y$, por lo tanto, su legitimiclad democrí 
tica sigue siendo discutible; pero, en este caso, ninguno de los regímenes de Europa Oriental sería legítimo. El problema no es éste. Aun en Bonn se insiste mayormente hoy dia en el hecho de que su legitimidad es discutible a causa cle la presencia de 20 divisiones soviéticas que permiten el mantẹnimiento del régimen. Pero tampoco es seguro que el régimen se derrumbe el día en que se retiren las tropas soriéticas. Sin duda, cambiará solamente de orientación y de personal. En el hecho, Alemania Oriental es el más próspero de los estados socialistas: es la segunda potencia industrial del bloque. Nadie puede negar, a menos que lo haga por educación o amistad, que posee un aparato estatal eficaz, con quien, por razones comerciales, conviene mantener relaciones de facto, las que a menudo son más importantes que las relaciones de julre que se tienen con ciertos otros estados oficialmente reconocidos. Aún más, para los países no europeos, Alemania Oriental no puede parecer menos honorable que ciertos países cuyos regímenes descansan visiblemente en la presencia militar extranjera.

Alemania Federal tiene, pues, cada yez más dificultades para mantener en vigor la doctrina Hallstein. Pero el fondo del problema no es jurídico: es una cuestión de principios políticos. Tiaj autodeterminación es uno de estos principios, sobre el cual, hasta el presente, Ja política francesa y ìa alemana han coincidido en lo que respecta a Alemania. Verdaderamente no se ha especificaclo cuál será la forma que ha de tomar esta autodeterminación, tanto que, a pesar de las apariencias, hay muchas soluciones posibles fuera de Ias elecciones libres a la manera occidental, siempre que estas soluciones sean imaginadas por los mismos alemanes y no solamente por las potencias extranjeras. De aquí el interés que presenta para el problema alemán, la orientación del actual gobierno, cuyos miembros than manifestado reiteradamente que están dispuestos a dar pruebas de su imaginación. Actualmente no podríamos anticiparnos a predecir su capacidad para realizar proyectos concretos; pero de todos modos podemos decir que algo está cambiando en Alemania. Como este renacimiento de la autonomía política alemana es absolutamente inseparable de las relaciones intereuropeas que se desenvuelven de una manera completamente pacífica, Alemania Federal, con o sin ejército, no puede thoy día conducir una diplomacia agresiva, ni más ni menos que cualquier otro país de Europa. Está obligada a tomar iniciativas sabias y mesuradas. Cualquier comparación con la época de entre-las-dos-guerras me parece anticientífica, aunque puede muy bien explicarse por sentimientos de temor, perfectamente comprensibles. 
Por el contrario, cualquier diplomacia alemana que se obstine en mantener una reivindicación, aunque sea implícita, sobre los territorios situados más allá de. la línea Oder-Neisse, me parece condenada al fracaso. Aunque no se ponja en tela de juicio el complicado aparato jurídico que sirve de fundamento a tal reivindicación, sería deseable que se acumularan declaraciones políticas formales en el senticlo inverso al que aparece en la nota alemana del año pasado sobre el desarme. En esta notá, preparada con intención conciliatoria, el gobiemo de Erhard reafirma la posición oficial y posterga el reconocimiento de la linea Oder-Neisse después de la firma de un tratado de paz que sobrevendría luego de la elección libre de un parlamento en toda Alemania, es clecir, después de la reunificación. Hasta este momento, según la posición oficial de Bonn, Alemania continúa existiendo en sus fronteras de 1937. Esta posición equivale a cerrarse, no solamente a toda posibilidad de acuerdo con Polonia, sino también con la Unión Soriética que ocupa, desde el fin de la guerra, una parte de la antigua Prusia Oriental, y para la cual esta posición alemana significa evidentemente que Bonn no desea, por ningún motivo, la reunificación.

Actualmente, todo el mundo está de acuerdo en que la reunificación alemana no se producirá como un hecho separado de la reunificación de Europa, y que esta última se hará, solamente, previa normalización. Por to tanto, el reconocimiento de todas las fronteras actuales es el acto político mínimo, inicial, al que Alemania Federal debe proceder para que su diplomacia ocupe el lugar que corresponde a su verdadera potencia.

La reunificación alemana no será otorgada por las grandes potencias. Será el fruto de la labor de los mismos alemanes? en la medicla en que sean capaces de encontrar la solución a sus problemas.

Para terminar, me referiré al nivel de relaciones bilaterales $y$ to haré con una nota optimista. Se trata, a la vez, de las relaciones polaco-escandinavas, anglo-checas, rumano-alemanas, etc., que constituyen la trama de la coexistencia pacífica en Europa. Se trata, igualmente, de las relaciones franco-soviéticas y de un tipo de relaciones entre Francia y los países de Europa Oriental que se ha desarrollado en el plano político en forma notoria desde hace algún tiempo.

Me limitaré a hacer dos advertencias acerca del crecimiento reciente de estas singulares relaciones:

1) Son inseparables de esta especie de renacimiento de la conciencia política nacional que, descle hace algunos años, apunta en la mayoría cle los países europeos, tanto al Este como al Oeste. 
2) Conducen al debilitamiento de las alianzas militares, pero en una forma muy especial; como si se tratara de las consecuencias de una negociación entre los dos grandes que nunca llegaron a efectuarse.

No se trata, de ninguna manera, de un retorno al sistema inestable de las alianzas europeas, que está definitivamente muerto desde la segunda guerra mundial, sino, probablemente, de la activa prepara; ción de una Convención Europea de Seguridad, prevista en el reciente comunicado franco-soviético, sin precisión de fecha.

En efecto, todas estas relaciones tienden a restablecer corrientes de intercambio y de contactos entre paises que siguen perteneciendo a sistemas más o menos distintos de integración económica y militar. La bipolaridad conserva sus derechos en Europa, lo que hace improbable y $\sin$ sentido la idea de un vuelco brusco de Alemania Occidental hacia el campo socialista o de una revolución prooccidental en Alemania Oriental, u otras imágenes catastróficas.

Tanto es así que el nacionalismo de que hemos hablado consiste más bien en una descentralización de la imaginación y de la iniciativa política que en un retorno a la completa independencia nacional, la cual carece de sentido en esta época de grandes espacios económicos. Lo que parece inevitable es que esta renovación más o menos desordenada, pero vigilada de cerca por los Grandes, con'duzca a toda Europa a la formación de una especie de zona de paz, ventajosa para todo el mundo.

Las negociaciones necesarias para la modificación de los dispositiros militares están hoy día subordinadas a esta voluntad política activa. Eso es una transformación radical si se recuerda que, en los tiempos de la guerra fria, la voluritad política fue constantemente subordinacla a la lógica de la confrontación militar. 